\title{
Contrast-enhanced ultrasound as support for prostate brachytherapy treatment planning
}

\author{
Bradley Pieters, MD, PhD, Prof. Hessel Wijkstra, PhD, Prof. Marcel van Herk, PhD, Ruud Kuipers, Emmie Kaljouw, MSc, \\ Prof. Jean de la Rosette, MD, PhD, Prof. Caro Koning, MD, PhD \\ Radiotherapy Department, Academic Medical Center/University of Amsterdam, Amsterdam, The Netherlands
}

\begin{abstract}
Purpose: To investigate the possibility of localization of intraprostatic lesions (IL) with contrast-enhanced ultrasound (CEUS) to support the brachytherapy treatment planning of temporary implants.

Material and methods: Two brachytherapy treatment plans were generated for 8 patients treated with external beam radiotherapy and pulsed-dose rate brachytherapy boost for prostate cancer. The first and second brachytherapy treatment plan was without and with knowledge of the localization of the ILs, respectively. Pairwise comparison was performed on prostate, rectum, and urethra dose-volume parameters and total reference air kerma (TRAK)-values.

Results: Coverage of the ILs by the $140 \%$ isodose was increased from mean $66.0-67.7 \%$ for the standard plan to mean 92.5-95.7\% for the adapted plan. The mean D90 of the ILs increased from 1.49-1.57 Gy/pulse to 1.76-1.81 Gy/pulse. Dosevolume parameters for the prostate, rectum, and urethra and the TRAK did not change.

Conclusions: CEUS technique is a promising method for IL localization to aid in brachytherapy treatment planning. Dose coverage on the IL could be improved without any increase of dose in organs at risk.

Key words: contrast-media, ultrasonography, image-processing, brachytherapy planning, prostate.

\section{Purpose}

Histologic examination of the prostate harboring a malignancy often reveals multiple areas with malignant lesions [1]. These malignant lesions differ in size and morphologic appearance and can be divided in dominant lesions and smaller insignificant lesions [2,3]. If radiotherapy is used for treatment it is a common practice to treat the whole prostate. In general, the dose to the prostate is evenly distributed with the same dose applied to malignant lesions as to healthy prostate areas. Van Lin et al. proposed identifying dominant intraprostatic lesions (DILs) better in order to treat the entire prostate to a moderate dose and giving a boost on these DILs [4]. To apply a boost dose to DILs, imaging techniques are needed to identify these areas. Several techniques are reported as able to identify tumor lesions within the prostate $[5,6]$. One of these techniques is Magnetic Resonance Imaging (MRI). T2-weighted imaging (T2-WI) provides a morphological localization of tumor lesions. T2-WI can be combined with functional imaging for additional information on tumor presence. For the purpose of functional imaging, dynamic contrast-enhanced MRI (DCE-MRI), diffusion-weighted imaging (DWI), and magnetic spectroscopic imaging (MRSI) can be used. There is increased evidence that the so-called multiparametric MRI (mpMRI), in which different sequences and combination of morphological and functional MRI are used, will increase the dia- gnostic accuracy for detection, localization, and characterization of prostate cancer. However, at the European Consensus Meeting in December 2009 it was clear that there is a large disagreement between experts in the field on criteria to standardize the conduct and interpretation of mpMRI [7]. Although very promising, for the above-mentioned reasons mpMRI still cannot be regarded useful for the routine clinical use [8]. Positron emission tomography (PET) lacks the high spatial resolution MRI has. Combination of PET with computer tomography (CT) offers better depiction of tumor areas within the prostate. Promising radiotracers are carbon- $11\left({ }^{11} \mathrm{C}\right.$-choline and ${ }^{11} \mathrm{C}$-acetate) and fluoro-18 $\left({ }^{18} \mathrm{~F}\right.$-fluorocholine and ${ }^{18} \mathrm{~F}$-fluoroacetate) [9].

Another modality to visualize tumor lesions is use of ultrasound (US) imaging. Particularly contrast-enhanced ultrasound (CEUS) imaging has the ability to detect these lesions [10]. This technique visualizes perfusion within the prostate. Because of increased microvessel density in tumor areas, discrimination with healthy prostate tissue is obtained. US contrast is provided by small encapsulated intravenously injected gas bubbles, which behave as additional reflectors in the blood stream. In a correlation study with prostatectomy specimens, Sedelaar et al. observed contrastenhanced power Doppler ultrasonography able to find up to $79 \%$ of lesions larger than $5 \mathrm{~mm}$ [11]. Modern contrast imaging technique uses the non-linear behavior of the mi- 
crobubbles to increase selective imaging of the bubbles for tumor detection [12]. Although in the early studies on CEUS for prostate cancer detection, low sensitivity and specificity rates were reported, modern ultrasound techniques and quantitative ultrasound imaging are more promising [11,13-15]. The recent reports indicating high accuracy for prostate cancer detection enable the CEUS technique to be used for prostate cancer radiotherapy $[16,17]$. Modern transperineal prostate brachytherapy is in general performed with US guidance. Brachytherapy is characterized by a heterogeneous dose distribution and it should be possible to treat the whole prostate gland to an elective dose with a boost on macroscopic tumor areas. The aim of this study was to investigate if CEUS techniques can support brachytherapy treatment planning. Since the value of the CEUS technique for routine clinical use has not been determined yet, this study should be considered as a proof of principle for future use.

\section{Material and methods}

Ten patients with prostate cancer who underwent external beam radiotherapy with pulsed dose-rate (PDR) brachytherapy were included in this study. The study is registered with www.trialregister.nl (number NTR1168) Details of the treatment were previously reported $[18,19]$. In brief, patients were treated for the prostate and base of the seminal vesicles with 3-dimensional conformal external beam radiotherapy to deliver a dose of $46 \mathrm{~Gy}$ in daily 2 Gy fractions. Subsequently, within 1 week a transperineal implantation was performed with flexible catheters for PDR brachytherapy. The Oncentra Prostate planning system (Nucletron B.V., Veenendaal, The Netherlands) was used for intra-operative treatment planning. Catheter positions were determined for provisional treatment planning. Definitive treatment planning was done with CT-scan without the rectal US probe to closely simulate the actual treatment geometry. The prescribed brachytherapy dose on the planning target volume (PTV) was $28.8 \mathrm{~Gy}$ in $1.2 \mathrm{~Gy}$ pulses and a time of 2.0 hours between pulses. The PTV was defined as the prostate gland without margin.

\section{CEUS image processing and identification of intraprostatic lesions}

Prior to start with external beam radiotherapy patients underwent an examination with CEUS as an outpatient procedure. The sulphur hexafluoride microbubbles (Sonovue $^{\mathrm{TM}}$ from Bracco, Milan, Italy) were injected intravenously via the antecubital fossa. US imaging was performed using an iU22 US scanner (Philips Healthcare, Bothel, USA) and a C8-4v endocavity probe. The power modulation technique was used for CEUS imaging. All imaging sequences were stored in the DICOM (Digital Imaging and Communication in Medicine) format, and transferred to a personal computer for further analysis. Off-line, the intraprostatic lesions in the CEUS sequences were delineated. This contouring was done in the transverse plane on areas with a fast or increased enhancement as compared to the main enhancement of the peripheral zone. In total, 2 boluses of $2.4 \mathrm{ml}$ of contrast were injected, and the inflow of contrast was monitored. During up to 4 minutes after the injection additional planes were examined using the destructionreplenishment technique. In this manner, the whole prostate was examined for suspected lesions. Imaging was performed and stored in the transverse orientation with a continuous grayscale sweep from base to apex, together with one longitudinal maximum cross-section. The midline of each frame in the transverse sweep is also located in the longitudinal cross-section. Using correlation techniques we were able to correlate each plane in the transverse sweep to one location in the longitudinal cross-section, and 3-dimensional (3D) reconstruction of the prostate was made. Each CEUS recording in a transverse plane was searched for in the recorded sweep, and therefore the location of each CEUS recording could be reconstructed into the 3D data set. The delineated lesions were drawn in this 3D data set, and the result was used for the fusion with the planning system imaging data set.

\section{Image fusion}

The US images used for brachytherapy planning were acquired using a $2 \mathrm{D}$ side viewing US probe which position was controlled by a stepper. The images had a resolution of $0.4 \times 0.4 \mathrm{~mm}^{2}$ in-plane with $1 \mathrm{~mm}$ slice separation. The brachytherapy planning images were combined into a $3 \mathrm{D}$ volume and registered to the $3 \mathrm{D}$ reconstructed US scan as described above. This data set had $0.17 \times 0.17 \times 0.17 \mathrm{~mm}^{3}$ voxels. Besides the reconstructed US scan, a corresponding 3D data set was available in which the CEUS detected intraprostatic lesion (IL) was indicated as binary mask. All 3D data sets were loaded into an in-house software system for image registration and fusion [20]. The software was extended with a rigid registration method suitable for US-US registration similar to the method proposed by Roche et al. [21]. First, both US images were manually aligned for 3D translation and rotation (6 degrees of freedom). During this step both US data sets were visualized in 3 orientations (transverse, sagital and coronal) and overlaid in a green-purple color wash or as an interactive cut display. Then, the US volumes were processed by an unsharp mask filter to extract gradient information. The final registration was performed by optimizing the correlation ratio metric between both processed US data sets. Both for visualization and registration purposes, the US scans were resampled using trilinear interpolation. The registration accuracy was mainly limited by differences in deformation between both US data sets because of difference in applied probe pressure. For the visual verification the registration accuracy was estimated to be better than $2 \mathrm{~mm}$. After approval of the registration, the binary volume with the delineated ILs was overlaid on the brachytherapy US using the $3 \mathrm{D}$ translation and rotation obtained from the US registration (Fig. 1).

A new treatment plan was created on the fused images. Treatment planning was performed with the Oncentra Prostate planning system. For each patient two plans were generated. The first plan without and the second plan with the ILs visualized. Dose constraints were formulated for the first treatment plan. The prostate volume covered by the reference dose (RD) should be $95 \%$ or more (V100-p $\geq 95 \%$ ), the minimal dose to the $2 \mathrm{ml}$ rectal volume receiving the highest dose should be $0.97 \mathrm{~Gy} /$ pulse or less (D2ml-r $\leq 0.97 \mathrm{~Gy} /$ 

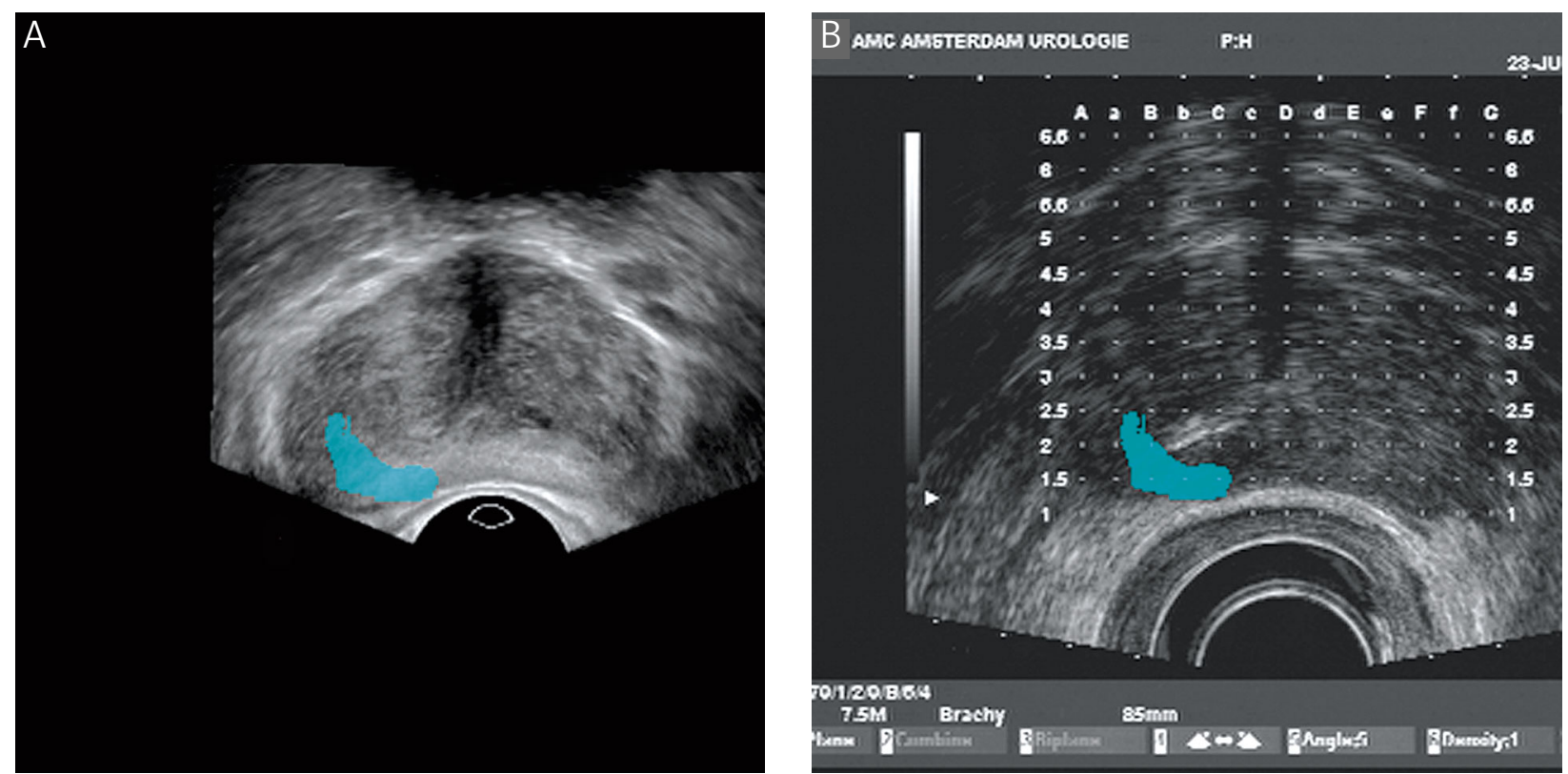

Fig. 1. Registered images of CEUS (A) and brachytherapy planning US (B) with delineated intraprostatic lesion

pulse), the maximum urethral dose (Dmax-u) should be $140 \%$ RD (1.68 Gy/pulse). The second treatment plan was created with the above-mentioned constraints and an additional constraint prescribing at least $95 \%$ of the IL volume covered by the $140 \%$ RD (V140-il $\geq 95 \%$ ) (Fig. 2).

The brachytherapy treatment plans with and without US contrast were compared to each other. Cumulative dosevolume histograms (DVH) were calculated and compared. DVH-parameters used for comparison were V100-p, V150-p, D90-p, D2ml-r, Dmax-u, and total reference air ker$\mathrm{ma}$ at $1 \mathrm{~m}$ (TRAK).

\section{Statistics}

Pairwise comparison of the means of the DVH-parameters was done by the Wilcoxon signed rank sum test. All tests were two-sided, and $P$-values $<0.05$ were considered significant. Statistical analysis was performed with the Pre- dictive Analytics SoftWare Statistics, version 18.0 for Mac OS X (PASW 18.0, Chicago, IL, USA).

\section{Results}

One patient did not show ILs on CEUS and for another patient fusion of CEUS-images on the brachytherapy treatment planning images was not possible due to a large variation in the prostate shape of the two studies. These two patients were excluded from further analysis. The median prostate volume was $35.9 \mathrm{ml}$ (range 16.8-50.2). Four patients had only 1 IL and the other 4 had 2 ILs at both sides of the prostate. The largest of both ILs within a prostate was coded as IL1 and the smallest - as IL2. Six of these ILs showed extracapsular extension (Table 1).

Table 2 shows that the mean V140 of the ILs was increased from $66.0-67.7 \%$ to $92.5-95.7 \%$. Also, the mean D90 on

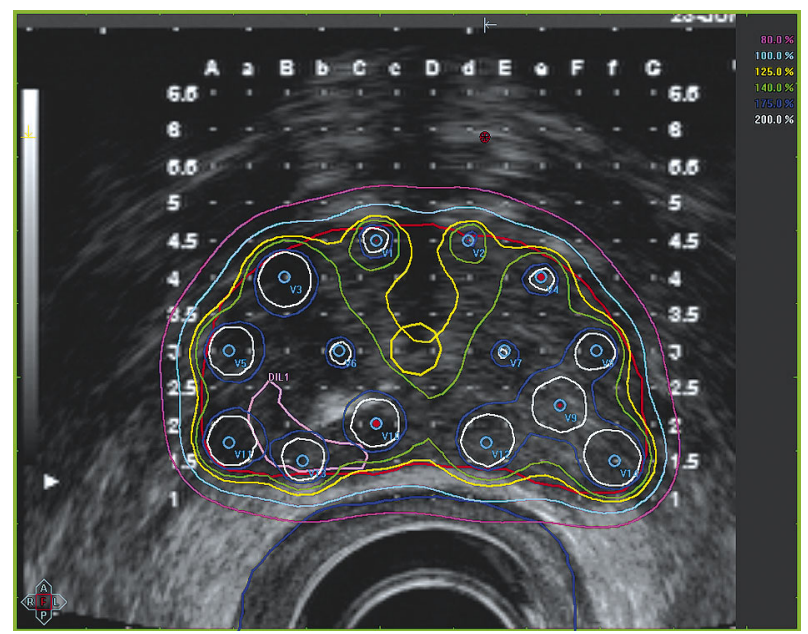

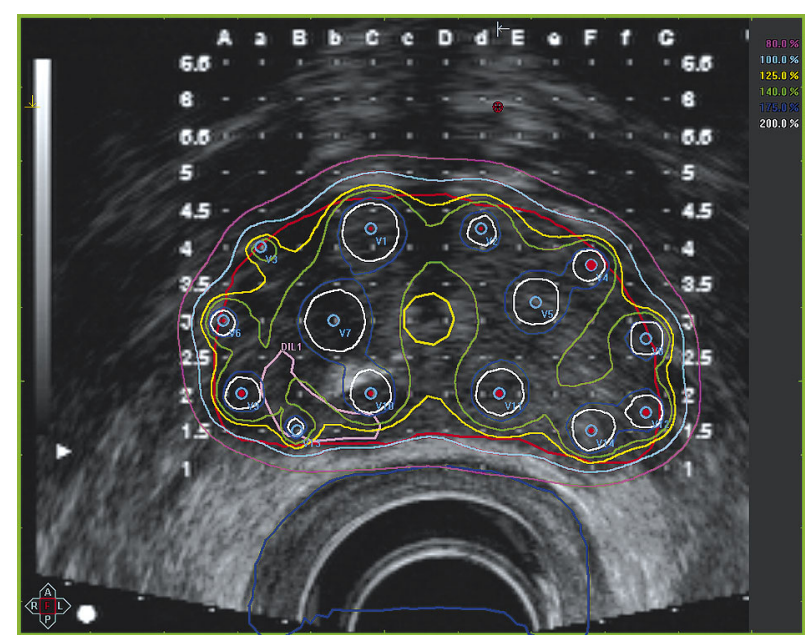

Fig. 2. Brachytherapy isodose distribution without (1A) and with (1B) knowledge of the position of an intraprostatic lesion in the right peripheral zone. Note the coverage of the IL by the $140 \%$ isodose 
Table 1. Intraprostatic lesion (IL) characteristics

\begin{tabular}{lcc} 
Prostate volume & median $35.9 \mathrm{ml}$ & range 16.8-50.2 \\
\hline $\begin{array}{l}\text { Number of ILs } \\
1\end{array}$ & 4 & \\
\hline 2 & 4 & \\
\hline \begin{tabular}{l} 
Extraprostatic extension \\
\hline Yes
\end{tabular} & 6 & \\
\hline No & 6 & \\
\hline Volume IL1 & median $0.25 \mathrm{ml}$ & range 0.04-0.53 \\
\hline Volume IL2 & median $0.09 \mathrm{ml}$ & range 0.03-0.18 \\
\hline Size IL1 & median $12.9 \mathrm{~mm}$ & range 6-18.1 \\
\hline Size IL2 & median $8.5 \mathrm{~mm}$ & range 5.9-11.0
\end{tabular}

the ILs was increased from 1.49-1.57 Gy/pulse to 1.76$1.81 \mathrm{~Gy} /$ pulse. In 4 cases, the first treatment planning was performed with 12 catheters and in the other 2 cases, 14 catheters were used. In all cases the same number of catheters was used by repositioning the catheters corresponding to the location of the ILs. The adapted treatment plan did not lead to an alteration of the DVH-parameters of the prostate, rectum, and urethra (Table 3). Also, a statistically non-significant increase of the TRAK was observed $(P=0.09)$.

\section{Discussion}

In this pilot study we have investigated the possibility of introducing CEUS data for prostate brachytherapy treatment planning. To our knowledge, this is the first study investigating this technique for brachytherapy. It appeared that with knowledge of the position of ILs, a better treatment plan could be generated with improved coverage of the ILs in the high dose regions. Better coverage of ILs was achieved by including the intraprostatic part as well as the

Table 2. Intraprostatic lesion (IL) mean dose-volume parameters for a non-adapted and adapted treatment plan

\begin{tabular}{lcc} 
& Non-adapted & Adapted \\
\hline V140 IL1 (\%) & 67.7 & 92.7 \\
\hline D90 IL1 (Gy/pulse) & 1.57 & 1.81 \\
\hline V140 IL2 (\%) & 66.0 & 95.7 \\
\hline D90 IL2 (Gy/pulse) & 1.49 & 1.76
\end{tabular}

Table 3. Mean dose-volume parameters and TRAK for a non-adapted and adapted treatment plan

\begin{tabular}{lccc} 
& Non-adapted & Adapted & $P$-value \\
\hline V100 prostate $(\mathrm{ml})$ & 35.7 & 35.8 & 0.40 \\
\hline V150 prostate $(\mathrm{ml})$ & 13.7 & 14.2 & 0.61 \\
\hline D90 prostate $(G y /$ pulse) & 1.36 & 1.37 & 0.61 \\
\hline D2ml rectum $(G y /$ pulse) & 0.84 & 0.88 & 0.08 \\
\hline Dmax-u (Gy/pulse) & 1.51 & 1.49 & 0.06 \\
\hline TRAK $(\mu$ Gy at $1 \mathrm{~m})$ & 459 & 473 & 0.09
\end{tabular}

extraprostatic extension into the high-dose region. The better coverage of the ILs resulted also in a higher dose (D90) in the ILs. In this small study we could not find an alteration in DVH-parameters of the prostate. What is more important, the adapted plans did not lead to higher doses in the rectum and urethra. We have succeeded by just repositioning the catheters to improve the adapted plan. Repositioning of catheters was done on inspection of the dose distribution in order to cover the ILs adequately. The distance for repositioning of a catheter was usually not more than 5 -mm compared to the original plan. We have not encountered any need for adding more catheters in our study, but that may be necessary in certain situations. Considering repositioning of catheters one should realize that this was a virtual repositioning. In a real implant the catheters can still end up in another position with an implication on the DVH-parameters. However, we belief that with knowledge of localisation of ILs it will influence the positioning of catheters to cover them optimally.

The TRAK was calculated to investigate changes in exposure to radioactivity. A statistically non-significant increase in TRAK was observed in the adapted plans compared to the original plans. Careful attention should be paid to TRAK values to be informed on exposure to radioactivity, which cannot be found in the prescribed dose or dose coverage to organs. Adaptation of treatment plans can easily lead to large differences in radiation exposure and should be considered in future studies.

The question still remains how accurate the CEUS technique is for identification of tumor lesions in order to introduce this concept with brachytherapy into the clinic. Halpern et al. compared the results of CEUS with 12 prostatectomy specimens [14]. In this small study they found a positive predictive value for tumor identification of only $56 \%$ and a sensitivity of $42 \%$. These numbers indicate a high false negative rate because tumor lesions are missed. In a similar study, Sedelaar et al. found CEUS investigation by an experienced investigator to have a detection rate of $61 \%$ for prostate tumor lesions [11]. However, they also found a detection rate of $79 \%$ for large sized $(\geq 5 \mathrm{~mm})$ lesions. The implication for brachytherapy is that at least the large sized tumor areas, clinically significant lesions, are identified. These high-volume tumor bearing areas probably need the highest dose [22].

A new development in the use of CEUS for prostate cancer detection is the ultrasound signal analysis and its quantification. The aim is to make the analysis less subjective and to increase the accuracy for tumor detection. At the Eindhoven University of Technology and Academic Medical Center, a diffusion parameter was investigated and compared to prostatectomy specimens [17]. A high sensitivity $(81.2 \%)$ and specificity $(84.6 \%)$ value was found for prostate cancer detection. Also the ROC area (Receiver Operating Characteristic area) under the curve was as high as 0.909 . This finding is very promising and needs further investigation. Other means of detecting malignant prostatic lesions with high spatial resolution is the use of MRI. However, also with the use of MRI tumor lesions can be missed or tumor extension can be misdiagnosed. In a systematic review with meta-analysis Engelbrecht et al. found, using ROC-curves, a test accuracy for discriminating $\mathrm{T} 2$ from T3 tumors of $71 \%$ 
[23]. In this study, a classification of T-stage was done on a per-prostate level and probably the accuracy on a per-lesion level would have been lower. At a European consensus meeting, there was a large disagreement between experts in the field on how to use mpMRI for prostate cancer imaging [7]. Consensus was reached on $67 \%$ of items related to imaging parameters for tumor detection and localization, and on only $54 \%$ of items related to imaging interpretation and reporting. So, even for the more clinically used imaging technique of MRI, as compared to CEUS, further investigation should be performed as to its validity. It is expected that both imaging techniques will play a major role in the future for image guided and targeted radiotherapy [24]. Because in general prostate brachytherapy, implantations are performed under US guidance, identification of tumor lesions on US is more advantageous than on MRI. If MRI is used for tumor localization purposes, fusion of images must be used if the US technique is used for implantation bringing in another source of geometric uncertainties. New developments in MRI-based implantations may circumvent this problem [25-28].

In our study we used rigid matching for contrast-enhanced images and US images for treatment planning. Because of shape deformation we encountered some difficulties in fusion of image studies. This uncertainty may question the reliability of this procedure in a clinical setting. For this reason, patients were not actually treated with the adaptive plan. However, we have shown as a proof of principle that by identification of tumor lesions within the prostate brachytherapy treatment plans can be adapted for better lesion coverage without any increase of dose in the OAR. CEUS is a promising technique to aid in adaptation of brachytherapy treatment plans. For practical clinical usage a suitable side-viewing probe should be developed for both contrast-enhanced images and transperineal implantations. After analysis of the images, contouring of the prostate and ILs is performed for brachytherapy planning. This way implantation is performed without the need for image fusion. Without such a dedicated probe, deformable image fusion techniques are absolutely needed for proper localization and positioning. When the treatment planning is done the implantation can be performed according to the plan and needle placement in the vicinity of the ILs.

\section{Conclusions}

Identification of ILs in the prostate with CEUS can help with adapting brachytherapy treatment planning for an improved coverage of the ILs without increasing the dose to the OARs. Further developments in image fusion and US hard- and software are needed before introduction into the clinic.

\section{References}

1. Villers A, McNeal JE, Freiha FS et al. Multiple cancers in the prostate. Cancer 1992; 70: 2313-2318.

2. Epstein JI, Walsh PC, Carmichael M et al. Pathologic and clinical findings to predict tumor extent of nonpalpable (stage T1c) prostate cancer. JAMA 1994; 271: 368-374.

3. Stamey TA, Freiha FS, McNeal JE et al. Localized prostate cancer. Cancer 1993; 71: 933-938.
4. van Lin ENJT, Fütterer JJ, Heijmink SWTPJ et al. IMRT boost dose planning on dominant intraprostatic lesions: Gold marker-based three dimensional fusion of CT with dynamic contrast-enhanced and ${ }^{1} \mathrm{H}$-spectroscopic MRI. Int J Radiat Oncol Biol Phys 2006; 65: 291-303.

5. Fuchsjäger M, Shukla-Dave A, Akin O et al. Prostate cancer imaging. Acta Radiol 2008; 1: 107-120.

6. Puech P, Huglo D, Petyt G et al. Imaging of organ-confined prostate cancer: functional ultrasound, MRI and PET/computed tomography. Curr Opin Urol 2009; 19: 168-176.

7. Dickinson L, Ahmed HU, Allen C et al. Magnetic resonance imaging for the detection, localisation, and characterisation of prostate cancer: recommendations from a European Consensus Meeting. Eur Urol 2011; 59: 477-494.

8. Heidenrich A. Consensus criteria for the use of magnetic resonance imaging in the diagnosis and staging of prostate cancer: not ready for routine use. Eur Urol 2011; 59: 495-497.

9. Jadvar H. Prostate cancer: PET with ${ }^{18} \mathrm{~F}-\mathrm{FDG},{ }^{18} \mathrm{~F}-$ or ${ }^{11} \mathrm{C}-\mathrm{ace}-$ tate, and ${ }^{18} \mathrm{~F}$ - or ${ }^{11} \mathrm{C}$-choline. J Nucl Med 2011; 52: 81-89.

10. Wink M, Frauscher F, Cosgrove D et al. Contrast-enhanced ultrasound and prostate cancer; a multicentre European Research Coordination Project. Eur Urol 2008; 54: 982-993.

11. Sedelaar JPM, van Leenders GJLH, Goossen TEB et al. Value of contrast ultrasonography in the detection of significant prostate cancer: correlation with radical prostatectomy specimens. The Prostate 2002; 53: 246-253.

12. Pallwein L, Mitterberger M, Pelzer A et al. Ultrasound of prostate cancer: recent advances. Eur Radiol 2008; 18: 707-715.

13. van Moerkerk H, Heijmink SWTPJ, Hulsbergen-van der Kaa CA et al. Computerized three-dimensional localization of prostate cancer using contrast-enhanced power Doppler and clustering analysis. Eur Urol 2006; 50: 762-769.

14. Halpern EJ, McCue PA, Aksnes AK et al. Contrast-enhanced US of the prostate with sonazoid: Comparison with wholemount prostatectomy specimens in 12 patients. Radiology 2002; 222: 361-366.

15. Seitz M, Gratzke C, Schlenker B et al. Contrast-enhanced transrectal ultrasound (CE-TRUS) with cadence-contrast pulse sequence (CPS) technology for the identification of prostate cancer. Urol Oncol 2011; 29: 295-301.

16. Mitterberger M, Aigner F, Pinggera GM et al. Contrastenhanced colour Doppler-targeted prostate biopsy: correlation of a subjective blood-flow rating scale with the histopathological outcome of the biopsy. BJU International 2010; 106: 13151318.

17. Kuenen MPJ, Mischi M and Wijkstra H. Contrast-ultrasound diffusion imaging for localization of prostate cancer. IEEE Trans Med Imaging 2011; 30: 1493-1502.

18. Pieters BR, van der Grient JNB, Blank LECM et al. Minimal displacement of novel self-anchoring catheters suitable for temporary prostate implants. Radiother Oncol 2006; 80: 69-72.

19. Pieters BR, Geijsen ED, Koedooder K et al. Treatment results of PDR brachytherapy combined with external beam radiotherapy in 106 patients with intermediate- to high-risk prostate cancer. Int J Radiat Oncol Biol Phys 2011; 79: 1037-1042.

20. Wolthaus JW, van Herk M, Muller SH et al. Fusion of respiration-correlated PET and CT scans: correlated lung tumour motion in anatomical and functional scans. Phys Med Biol 2005; 50: 1569-1583.

21. Roche A, Pennec X, Malandain G et al. Rigid registration of 3-D ultrasound with MR images: a new approach combining intensity and gradient information. IEEE Trans Med Imaging 2001; 20: 1038-1049.

22. Korporaal JG, van den Berg CA, Groenendaal G et al. The use of probability maps to deal with uncertainties in prostate cancer delineation. Radiother Oncol 2010; 94: 168-172. 
23. Engelbrecht MR, Jager GJ, Laheij RJ et al. Local staging of prostate cancer using magnetic resonance imaging: a metaanalysis. Eur Radiol 2002; 12: 2294-2303.

24. de la Rosette J, Ahmed H, Barentsz J et al. Focal therapy in prostate cancer. Report from a consensus panel. J Endourol 2010; 24: 775-780.

25. Moman MR, van den Berg CAT, Kruger AEB et al. Focal salvage guided by $\mathrm{T} 2$-weighted and dynamic contast-enhanced magnetic resonance imaging for prostate cancer recurrences. Int J Radiat Oncol Biol Phys 2010; 76: 741-746.

26. Nguyen PL, Chen M, D'Amico AV et al. Magnetic resonance image-guided salvage brachytherapy after radiation in select men who initially presented with favorable-risk prostate cancer. Cancer 2007; 110: 1485-1492.

27. Ménard C, Susil RC, Choyke P et al. MRI-guided HDR prostate brachytherapy in standard 1.5T scanner. Int J Radiat Oncol Biol Phys 2004; 59: 1414-1423.

28. Macura KJ, Stoianovici D. Advancements in magnetic resonance-guided robotic interventions in the prostate. Top Magn Reson Imaging 2008; 19: 297-304 